\title{
Study of thermal properties of biodegradable composite materials based on recycled polypropylene
}

\author{
R. B. Salikhov ${ }^{\dagger}$ M. V. Bazunova, A. A. Bazunova, T. R. Salikhov, V. P. Zakharov \\ †salikhovrb@ya.ru
}

Bashkir State University, 32 Z. Validi str., Ufa, 450076, Russia

Recently, the development of modern technology for producing biodegradable polymers is one of the most topical issues. Filling the polymer with natural components increases the interfacial contact boundary, through which moisture and aggressive chemicals can penetrate into the material, which accelerates the decomposition of the composite in natural conditions. This study is devoted to the study of the thermophysical properties of biodegradable composite materials based on recycled polypropylene with various natural fillers of plant origin. In this work, we measured the thermal conductivity and resistivity depending on temperature, filler concentration and residence time in the recovered soil in which the samples were biodegraded, shows the relationship between the change in thermal conductivity and resistivity of the studied samples and proposed preliminary conclusions about the mechanisms of thermal conductivity of the materials considered. Adding natural fillers of plant origin to composites based on recycled polypropylene gradually increases the thermal conductivity coefficient with increasing filler content. In this case, the resistivity decreases, and, with an increase in the content of the filler, this decrease becomes more significant. This means that with an increase in conductivity, the coefficient of thermal conductivity also increases, which is typical for materials in which charge transfer occurs at the expense of electrons. As a result of destruction under the influence of the environment, the thermal conductivity of the samples decreases, and the change is most significant for samples with a high content of filler.

Keywords: biodegradable polymers, thermal conductivity, resistivity.

\section{Introduction}

It is known that, depending on the purpose and operating conditions, polymeric materials must meet the requirements for mechanical properties, thermal and chemical resistance, etc. [1]. At present, a new approach to the development of polymer materials is being formed, which consists in the need to obtain polymers that retain their performance only during the period of consumption, and then undergo physical, chemical and biological transformations under the influence of environmental factors and are easily included in the metabolism of natural biosystems [2-4]. Currently, biodegradable polymer composites based on large-capacity synthetic polymers and natural fillers of plant origin (wood flour, buckwheat husks, rice straw and other cellulosecontaining materials) are often used to create polymer products with regulated service life [5-8]. The filling of the polymer with natural components, including those of plant origin, increases the interface through which moisture and aggressive chemicals can penetrate the material, which accelerates the decomposition of the composite in natural conditions [9].

Polyolefins, such as polyethylene and polypropylene (PP), are one of the most common large-capacity polymers $[10,11]$. Accordingly, the urgency of solving the problem of waste disposal of products from these polymers is increasing. In connection with the above, as a binding component of biodegradable polymer composite materials, it is advisable to use recycled PP.

The introduction of fillers into polymers, including PP, leads to a change in both operational and technological properties of polymer composite materials based on them [12-14]. Recycling of PP and composites on its basis in the product provides, as with other thermoplastics, melting and cooling of the polymer. Accordingly, it is necessary to study the thermal properties, such as thermal conductivity, heat capacity, thermal conductivity, coefficient of linear thermal expansion, the knowledge of which allows us to calculate the temperature regime of molding products and determine the application of materials. $[15,16]$.

The aim of this work was to study the thermophysical properties of biodegradable composite materials based on secondary polypropylene with various natural fillers of plant origin, as well as the effect of biodegradation of these composites on the thermophysical properties. To achieve this goal, the thermal conductivity coefficient was measured depending on the temperature, filler concentration and the time spent in the reduced soil in which the samples were biodegraded, the relationship between the thermal conductivity coefficient and the specific resistance of the samples was shown. On the basis of the obtained results, preliminary conclusions about the mechanisms of thermal conductivity of the materials considered are proposed. 


\section{Experimental part}

In work samples of the recycled PP corresponding to $\mathrm{PP}$ of the $\mathrm{H}-350 \mathrm{FF} / 3$ brand (further - recycled-PP) representing the crushed material from the substandard products made by a method of molding under pressure in technological production of plastic products plant "Alternative» (the Republic of Bashkortostan, Oktyabrsky) were used.

As natural fillers of plant origin used pre-drained and crushed buckwheat husk (BH), provided by LLC «Magistral», with an average particle size of $0.5 \mathrm{~mm}$, containing $47-58 \%$ cellulose, $10-12 \%$ lignin, 5-7\% hemicellulose, the rest minerals, chaff $\mathrm{CH}$ ), provided by LLC "Davlekanovsky combine of bread products №2", with an average particle size of $0.15 \mathrm{~mm}$, containing $40-46 \%$ cellulose, $6-8 \%$ lignin, $24-32 \%$ hemicellulose, the rest-minerals and wood flour (WF) brand 180 needles (Dzerzhinsk), corresponding to GOST 16361-87 and TU 5386-001-87877379-2014, with an average particle size of about $0.17 \mathrm{~mm}$ and containing $45-52 \%$ cellulose, $35 \%$ lignin, $17-23 \%$ hemicellulose.

Preparation of polymer composites was carried out in the melt at the laboratory station (plastograph) "Plastograph EC" (Brabender, Germany) for 15 minutes at a load of $200 \mathrm{~N}$ at a temperature of $180^{\circ} \mathrm{C}$, followed by pressing on the automatic hydraulic press "Auto MH-NE" (Carver, USA) at $210^{\circ} \mathrm{C}$ and holding at a pressure of $7000 \mathrm{kgf}$ for 3 minutes. The dosage of vegetal filler was calculated in weight parts (wt. pts.) at 100 wt. pts. of PP.

To carry out the biodegradation of polymer materials samples, a soil test was carried out on the restored soil. The soil for testing was prepared from horse manure, garden soil and sand, taken in equal mass proportions. Polymer samples were loaded into boxes with soil vertically into the soil with subsequent exposure for 4 months at room temperature in the laboratory. Then the samples were extracted from the soil, cleaned from the soil and dried at a temperature of $(80 \pm 2)^{\circ} \mathrm{C}$. The degree of biodegradation of polymer composites samples based on recycled and natural fillers of plant origin after composting in soil is estimated by the weight loss of samples by weighing, carried out with a certain periodicity. The first indicators of mass loss were recorded after 30 days of placing in the reconstructed soil for samples with high degrees of filling ( $30-50$ parts by weight of vegetable filler). After 120 days after the start of the experiment, visual changes in the samples placed in the soil were not visible, but there was a loss of mass in all the studied samples, not exceeding $4 \%$.The method of measuring the thermal conductivity of composite materials based on recycled polypropylene with various natural fillers of plant origin is that the sample in the form of a disk is necessary to create a uniform stationary heat flow. This flow is created by an electric heating furnace located at one end of the sample. The other end has contact with the heat tank, which performs the functions of a massive copper blank. The amount of heat reported by the electric heater per unit time is equal to the furnace power $Q=I U$. Then the specific heat flux through the cross section of the rod: $q=k Q / s$, where $k-$ empirically selected coefficient, taking into account heat loss. Hence, $q=4 \mathrm{kIU} / \pi$. At the same time, the specific heat flux, according to the Fourier law, through the disk is: $q=\lambda \Delta T / l$, where $\lambda$ is the thermal conductivity coefficient of the rod material, $\Delta T$ is the temperature difference on the opposite sides of the disk thickness $l$. The temperature difference is determined by a thermocouple (copper-Constantan).

To obtain the absolute values of the thermal conductivity coefficient, it is necessary to measure the sample with a known (table value of thermal conductivity). As a reference sample material was chosen getinax (thin), used for the manufacture of electrical boards. Based on the table value $\lambda=0.23 \mathrm{~W} / \mathrm{m} \cdot \mathrm{K}$, it is possible to recalculate the results of the measurements and find the values of the thermal conductivity coefficient for different samples.

Measurements and installation for measurements were performed in accordance with GOST 23630.2-79 (method for determining thermal conductivity), which applies to plastics with thermal conductivity from 0.1 to $5 \mathrm{~W} / \mathrm{MK}$ and establishes a method for determining thermal conductivity in the temperature range from minus 100 to plus $400{ }^{\circ} \mathrm{C}$ (from 173 to $673 \mathrm{~K}$ ). Moreover, the height of the sample $(h)$ is selected depending on the expected value of the thermal conductivity coefficient $(\lambda)$ from Table 1.

To carry out the measurements, an experimental stand was assembled, the block diagram of which is shown in Fig. 1.

The error in determining the thermal conductivity coefficient was determined by the accuracy of measuring the temperature difference, the thickness of the sample and did not exceed $3 \%$.

\section{Discussion of results}

It is known that the thermophysical properties of the filled polymers depend on the proportion of the filler in the polymer composite material, the size and shape of the filler particles, the distribution of the filler in the matrix, the nature of the interaction of the filler with the polymer [17].

Table 1. The height values of the material sample, depending on the expected value of the thermal conductivity coefficient.

\begin{tabular}{|c|c|c|c|c|c|}
\hline$\lambda, \mathrm{W} / \mathrm{m} \cdot \mathrm{K}$ & $0.1-0.3$ & $0.3-0.5$ & $0.5-1.0$ & $1.0-2$ & $\begin{array}{c}\text { more } \\
\text { than } 2\end{array}$ \\
\hline$h \cdot 10^{3}, \mathrm{~m}$ & $0.5-1.0$ & $1.2-2$ & $2-3$ & $3-5$ & 5 \\
\hline
\end{tabular}

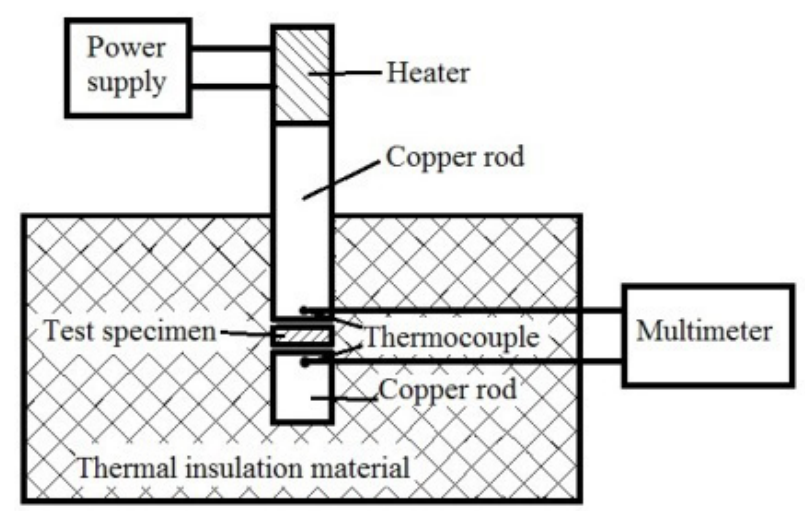

Fig. 1. Block diagram of the measuring stand for determining the thermal conductivity of materials. 
Measurements of the thermal conductivity coefficient showed that the values of this parameter for all studied samples of composite materials based on fluorine-PP and natural fillers of plant origin lie in the range of $0.10-0.17 \mathrm{~W} / \mathrm{m} \cdot \mathrm{K}$.

Graphs showing changes in the thermal conductivity of samples (Fig. 2) depending on the amount of filler, it is shown that the addition of buckwheat husk and chaff with an increase in the content of the filler is a gradual increase in the thermal conductivity coefficient.

As follows from the data shown in Fig. 3, when adding natural fillers of plant origin, the resistivity decreases, and, with increasing filler content, this decrease becomes more significant, except for samples with rice husk and wood flour.

Taking into account the presented results, it can be seen that in samples with a lower resistivity value, the thermal conductivity value is higher. This means that with increasing conductivity, the coefficient of thermal conductivity increases, which is typical for materials in which the charge transfer is carried out by electrons [18].

It is known that fillers that lead to improvement of mechanical properties of polymers, in the absence of moisture, have little effect on their electrophysical properties [19, 20]. The electrical conductivity of composite polymer materials is mainly determined by the electrical properties of the fillers and their dispersion, the structure of the polymer and the method of introducing fillers into the polymer. As shown by the research, the nature of the electrically conductive filler has a great influence on the electrical properties of the compositions. Most fillers reduce the resistivity only at concentrations of $40 \%$ by weight and higher. Such high concentrations make polymer compositions fragile and unsuitable for structural products. In the studied samples the concentrations did not exceed $30 \%$, no significant effect on the electrical conductivity was found. Similar conclusions can be drawn about the thermal properties and thermal conductivity.

There is no doubt that the process of biodegradation needs to influence the thermophysical properties-likernyh compositions. Therefore, before recommending new materials for practical application, it is necessary to investigate

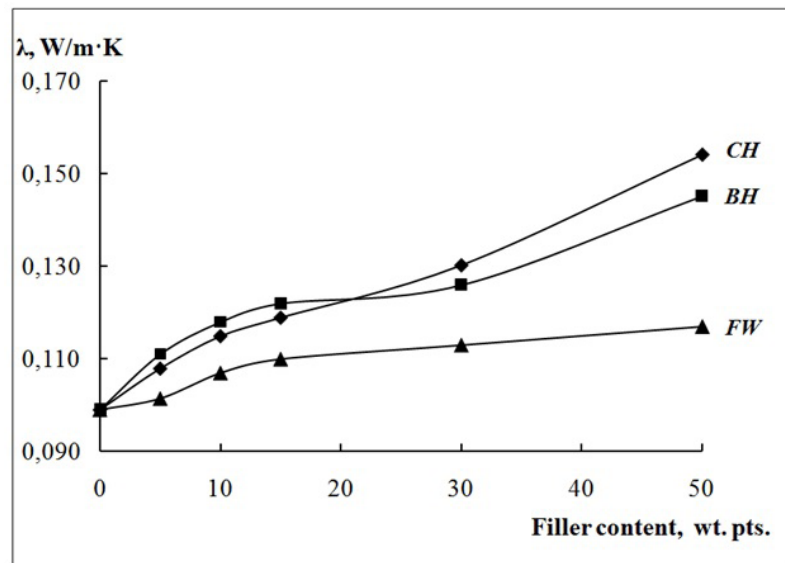

Fig. 2. The dependence of the coefficient of thermal conductivity of samples of composite materials based on the recycled-PP depending on the amount of vegetal filler. the change of their properties in the environment, including in contact with the soil.

As a result of biodegradation under the influence of soil microorganisms, the coefficient of thermal conductivity of samples decreases (see Fig. 4), moreover, the change is most significant for samples with a high content of vegetable filler. This fact can be explained by a higher degree of biodegradation of high-filled samples, in which the "leaching" of the filler and low molecular weight fractions from the polymer matrix of the composite increases the porosity in the structure of the composite, and, accordingly, the decrease in the thermal conductivity.

To generalize the results obtained, it is necessary to involve the currently known General ideas and regularities concerning the thermophysical properties of materials. Solids on the mechanism of heat transfer is divided into three groups [20]:

1) dielectrics, in which the thermal conductivity is due to lattice vibrations;

2) metals, for heat transfer in which electrons are mainly responsible;

3) poorly conducting substances, combining both types of thermal conductivity.

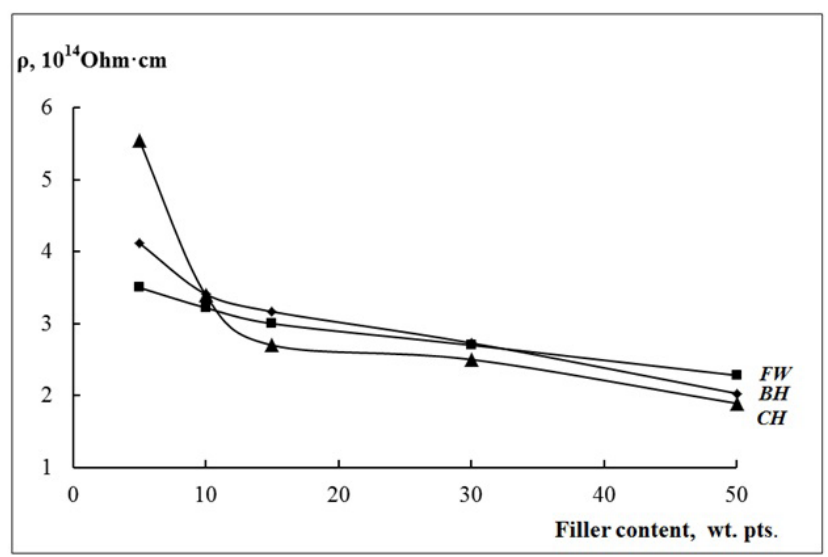

Fig. 3. Dependences of resistivity of samples of composite materials based on recycled -PP depending on the type of vegetal filler.

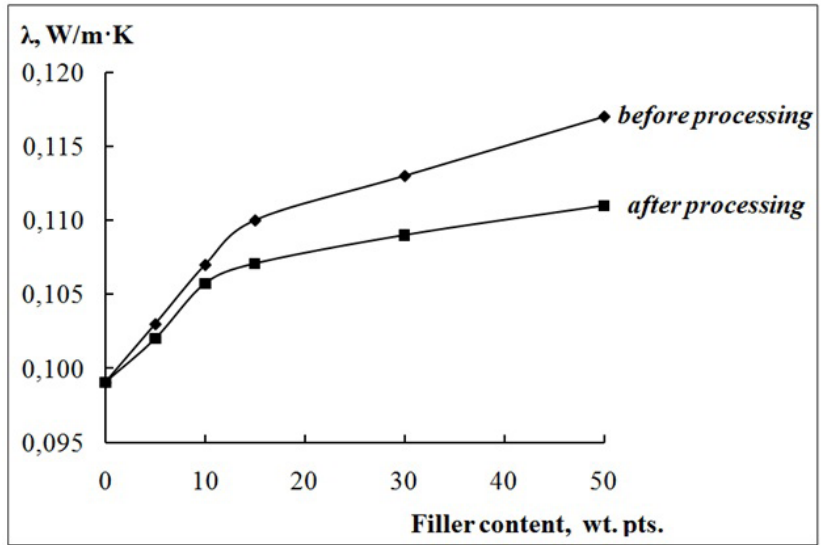

Fig. 4. Effect of biodegradation after exposure to reduced soil for 4 months on the thermal conductivity of samples of composite materials based on recycled -PP with different natural fillers of plant origin. 
The thermal conductivity of polymer samples was studied at temperatures close to room temperature (from 40 to $80{ }^{\circ} \mathrm{C}$ ). The purpose of these studies was to establish the dependence of the thermal conductivity of the composition and the number of fillers used. There was a slight increase in thermal conductivity with increasing temperature, which is typical for amorphous polymers.

The models considered in the literature are based on the "partial" contributions of the binder and randomly embedded filler particles. However, the experiment shows that not only the properties and relative content of individual components play an important role in the process of heat transfer in polymer composite materials. A certain role is played by the aggregation of filler particles, which leads, when the concentration reaches a certain threshold value, to the establishment of conducting "bridges" between the particles and the appearance of the hopping conductivity effect. It is necessary to take into account the modification of the physical properties of the polymer at the interface, due to the interaction of the binder with the surface of the filler particles. In addition, it is possible to loosen the binder, which determines the presence of the third component in the composition pores.

Thus, when creating biodegradable polymer composite materials, it is necessary to take into account that practically all polymer materials are exposed to biodegradation in the natural environment, but for most of them the rate of destruction is extremely low. It depends on the nature of the polymer, as well as the type of plasticizer and filler used in the manufacture of products. The rate of biodegradation of polymer materials also largely depends on the spatial availability of macromolecules to biological agents, which is determined by the hydrophilic-hydrophobic properties of the materials surface, their supramolecular organization and macrostructure [21-22]. There is no doubt that the filling of polymer materials with plant components not only significantly changes the rate of biodegradation, but, as shown in this work, due to changes in supramolecular organization and the macrostructure of the material affects on the thermophysical properties.

\section{Summary}

On the basis of the results obtained, the following main conclusions can be drawn. When natural fillers of plant origin are added to composites based on secondary polypropylene, the thermal conductivity coefficient gradually increases with the filler content. In this case, the resistivity decreases, and, with an increase in the filler content, this decrease becomes more significant, except for samples with rice husks and wood flour. This means that with increasing conductivity, the coefficient of thermal conductivity increases, which is typical for materials in which the charge transfer is carried out by electrons. As a result of destruction under the influence of the external environment, the coefficient of thermal conductivity of samples decreases, and the change is most significant for samples with a high filler content.
Aknowledgements. The article was prepared in the framework of research work in the Bashkir State University with the financial support of the Ministry of education and science of the Russian Federation (contract № 03. G25.31.0275).

\section{References}

1. V.K. Kryzhanovsky, V.V. Burlov, Yu. V. Panimatchenko, Yu. V. Kryzhanovskaya. Technical properties of polymeric materials: Uch.-sprav. pos. Saint Petersburg, Publishing house "Profession" (2003) 240 p. (in Russian)

2. A. Shan, F. Hasan, A. Hameed, et al. Biotechnol Fdv. 26, 246 (2008).

3. V.A. Fomin, V.V. Guzeev. Plastics. 2, 42 (2001). (in Russian)

4. S.Z. Rogovina. Polymer Science, Series C. 58, 62 (2016).

5. E. E. Mastalygina, N.N. Kolesnikova, A.A. Popov. Prospective materials. 9, 39 (2015). (in Russian)

6. E. E. Mastalygina, O.V. Shatalov, N.N. Kolesnikova, A. A. Popov, A. V. Krivandin. Inorganic Materials: Applied Research. 7(1), 58 (2016).

7. M.V. Bazunova, R.B. Salikhov, A.R. Sadritdinov, V.V. Chernova, V.P. Zakharov. Journal of Pharmaceutical Sciences and Research. 10(2), 288 (2018).

8. D. G. Dikobe. Express Polym Lett. 4(11), 729 (2010).

9. M. Rutkowska, A. Heimowska, K. Krasowska, et al. Polish Journal of Enviromental science. 11(3), 267 (2002).

10. A. N. Filonov, N. V. Maksimova, I. N. Knir, E. A. Mayer. Plastic masses. 5, 30 (2004). (in Russian)

11. V.G. Makarov, V.B. Koptenarmusov. Industrial thermoplastics: Handbook. Moscow, ANO Publishing House Chemistry, Kolos Publishing House (2003) 208 p. (in Russian)

12. Yu. S. Lipatov. Physical and chemical bases of filling polymers. Moscow, Chemistry (1991) 245 p. (in Russian)

13. M. V. Bazunova, V. V. Chernova, R. B. Salikhov, E. I. Kulish, V.P. Zakharov. Bulletin of the Bashkir University. 23(1) 70 (2018). (in Russian)

14. M.V. Bazunova, A.S. Vasyukova, R. B. Salikhov, V.P. Zakharov. Bulletin of the Technological University. 21(7), 37 (2018). (in Russian)

15. N.N. Teryaeva, O. V. Kasyanovva, T.V. Lopatkina. Bulletin of the Kuzbass State Technical University. 4(1), 69 (2005). (in Russian)

16. Y.K. Godovsky. Thermal physics of polymers. Moscow, Chemistry (1982) 280 p. (in Russian)

17. H. Essabir, E. Hilali, A. Elgharad, et al. Mater Design. 49, 442 (2013).

18. R. B. Salikhov, T. R. Salikhov. Letters on Materials. 5(4), 442 (2015). DOI: 10.22226/2410-3535-2015-4-442-447

19. M. F. Galikhanov, D. A. Eremeev, R. Y. Deberdeev. Russian Journal of Appl. Chem. 10, 1651 (2003).

20. V.G. Shevchenko. Fundamentals of Physics of Polymer Composite Materials. Moscow, MGU (2010) 99 p. (in Russian)

21. M.I. Shtilman. Journal of Siberian Federal University. Biology. 8(2), 113 (2015).

22. E. A. Kuznetsov. Applied ecobiotechnology. Moscow, Binom, Laboratory of Knowledge (2012) 629 p. (in Russian) 